\title{
Arbor
}

\section{La sombra del 98}

\author{
Manuel Espadas Burgos
}

Arbor CLXIX, 666 (Junio 2001), 371-381 pp.

El centenario de la crisis de 1898, sin duda una clara divisoria para una comprensión histórica de la España contemporánea, ha originado una amplia reflexión que se ha manifestado tanto en la forma de un mantenido debate historiográfico como en un estímulo de la investigación y en una copiosa y, como es de suponer, desigual producción bibliográfica. Debate historiográfico $\mathrm{y}$, sobre todo, nuevas investigaciones, desde la serena perspectiva que permite un horizonte de cien años, han modificado sustancialmente la visión que se tenía de aquella crisis y, sobre todo, han contribuido a superar y, en ocasiones, arrumbar algunos de los tópicos y de los clichés que se habían perpetuado a raiz de una particular apreciación de aquellos acontecimientos y de sus consecuencias, rebajando en gran medida los perfiles de un dramatismo histérico que, si en individualidades o en sectores muy concretos existió, no se podía atribuir a sectores mayoritarios y menos aún al conjunto de la sociedad española.

1. El Madrid de 1898 es, esencialmente, el Madrid de la Restauración, una ciudad que tanto en lo demográfico como en lo urbanístico se encontraba en claro proceso de transformación y de superación de los caracteres, aún muy propios de antiguo régimen, que la habían definido durante la monarquía isabelina. En lo demográfico, es cierto que aun a fines de siglo Madrid pudiera todavía tenerse como una ciudad donde los problemas alimentarios, la situación de la higiene pública y los frecuentes brotes epidémicos, como de forma muy aguda había sido el cólera de 1885 , se mostraban como causas de una adversa realidad demográfica ${ }^{1}$. Y aunque la población madrileña creciese en números absolutos, la consulta de los censos de esos años finales del siglo muestran cómo el número de los nacidos fuera de la Villa superaba 
al de nacidos en Madrid. Precisamente la inmigración iba a ser uno de los factores más importantes de crecimiento del Madrid finisecular, primer foco nacional de atracción migratoria, empezando naturalmente por la procedente de las provincias limítrofes, dirigida especialmente hacia el sector de los servicios. El año que cierra el siglo, la población de Madrid es de 539.835 habitantes, de los cuales un 50\% habían nacido fuera de Madrid. Con Barcelona, también importante foco de atracción migratoria, Madrid marcaba la tendencia que caracterizaría a la población española del fin de siglo, el crecimiento de las zonas urbanas, de los municipios con más de diez mil habitantes en detrimento de la España rural y con tasas muy superiores a la media de crecimiento demográfico nacional.

Son los años en que, como consecuencia de ese aumento de población, los problemas de la vivienda se agudizan y se convierten en tema de preocupación para gobernantes y partidos políticos. Son los años del ensanche de Madrid, al norte con la expansión de los distritos de Hospicio y Universidad; al sur, por el distrito de Hospital, creándose una zona de ocupación de proletariado industrial que venía a sumarse a la anterior población artesanal situada en torno al eje de la calle de Atocha. Al tiempo, los distritos de Latina e Inclusa iban albergando una también creciente población obrera, mientras el distrito de Centro se convertía en el núcleo comercial y burocrático de la ciudad. Ya en 1883 había escrito La Epoca: "Ya ha pasado Madrid de la Castellana por el norte; de la estación de Atocha por el sur; de la plaza de toros por el este y de Chamartín por el oeste». El de Madrid coincide con el «ensanche» de otras ciudades españolas, consecuencia del crecimiento demográfico, de la diversificación de funciones sociales, del desarrollo industrial y de unos nuevos modelos urbanísticos. Son los años en que se trazan las nuevas avenidas, generalmente sobre los ejes históricos de la ciudad o bien, abriendo otros nuevos, la época en que se introducen los estilos arquitectónicos y decorativos que triunfan en Europa, pero también aquella en que se resucitan viejos estilos que se consideran representativos de un "arte nacional» enraizado con el ser histórico de España. Así, Madrid se llena de «neogóticos» y de «neomudéjares», tanto en edificios religiosos como civiles.

La «Gran Via», sobre proyecto del arquitecto municipal Carlos Velasco Peinado aprobado en 1887, es el símbolo de ese nuevo Madrid que cruza la frontera de 1900 , si bien hasta 1910 no se inaugurasen oficialmente las obras, que habían supuesto, entre otras cosas, el obligado derribo de 339 inmuebles. Sin duda, el otro modelo de la transformación urbanística de Madrid sería «la ciudad lineal», reflejo en 
España de otros modelos europeos de «ciudades lineales», que entroncaba de un lado con el crecimiento demográfico de Madrid pero, de otro, con los planteamientos de una nueva conciencia social. En el pensamiento de su creador, Arturo Soria, muy representativo de la mentalidad propia de los hombres de la Institución Libre de Enseñanza y de la recién creada Comisión de Reformas Sociales, estaba la idea de que muchas de las lacras sociales que vivía la época, entre ellas la violencia o la delincuencia, desaparecerían en cuanto el hombre se sintiese situado en un ambiente menos agresivo. "Para cada familia una casa. En cada casa, una huerta y jardín» era el lema del proyecto de aquella nueva ciudad, convencido Soria de que «hacer una nueva ciudad es mucho mejor y más barato que remendar una vieja», con lo cual criticaba el polémico trazado de la proyectada Gran Via.

No sería solamente la "ciudad lineal» la respuesta a los problemas de vivienda. Los últimos años del siglo abren una política de «casas baratas», ejemplo de la cual serían entidades como "La Constructora Benéfica", que levantaría un reducido número de viviendas en los barrios de Pacífico y de Cuatro Caminos; o «El Porvenir del Artesano», una constructora mútua que edificó junto a la carretera de Francia. Muy a comienzos del nuevo siglo, la celebración en varios países europeos de congresos internacionales de casas baratas tuvo especial incidencia en España. Fruto de ello fue la creación en 1905 del Servicio de Empadronamiento Sanitario de las Viviendas, fuente de información a partir de la cual el Ayuntamiento pudo conocer en qué condiciones se encontraban las barriadas más pobres y abandonadas.

La Puerta del Sol seguía siendo el corazón de la ciudad. A los extranjeros que visitaban Madrid les asombraba su bullicio, su contínuo ir y venir de gentes. Uno de ellos, el ruso Nemirovich - Dancheko, escribía en 1898: «Aquí se entera uno de las noticias, se hacen amistades, aquí nace y crece el chisme» ${ }^{2}$.

Desde el punto de vista de su composición social, la población de Madrid la seguían formando una nobleza mezcla de los restos de la vieja aristocracia del Antiguo Régimen y de la nueva oligarquía surgida al hilo del asentamiento del régimen liberal, junto a una ascendente clase media estratificada de industriales, negociantes, especuladores, funcionarios militares y civiles, y unas amplias clases populares, con un artesanado degradado, heredero de los antiguos gremios, un amplio componente dedicado al servicio doméstico y un incipiente proletariado industrial. Los intereses coloniales habían desempeñado un importante papel en el proceso de ascenso y asentamiento social de la burguesía española, en este caso, de la madrileña, a los que tampoco fueron 
ajenos los intereses del estamento nobiliario madrileño. Pongamos los casos, entre otros, del marqués de Vinent, ligado al Banco de Castilla y al Hispano Colonial; de don Juan Manuel de Urquijo, agente de cambio y bolsa, para terminar convertido en cabeza de una de las bancas más importantes del Madrid contemporáneo; o del marqués de Manzanedo y duque de Santoña, quizá el hombre más representativo de los intereses esclavistas coloniales, que llegaría a ser el primer contribuyente madrileño por propiedad urbana; o, por último, de don Antonio López y López de Lamadrid, primer marqués de Comillas, el promotor y propietario de la compañía «Trasatlántica» que aseguraba el transporte, sobre todo el de tropas, con Ultramar.

Pero, sobre todo, el Madrid que traspasa el cambio de siglo es el Madrid de funcionarios y menestrales, el que admirablemente recogieron algunos de los «Episodios Nacionales» de Galdós o describieron plumas como Pedro de Répide, Emiliano Ramirez Angel, Emilio Carrere o Ramón Gómez de la Serna; el Madrid popular y provinciano que frecuentaba los teatros, que se emocionaba con el «Juan José» de Joaquín Dicenta, estrenada en octubre de 1895, una obra de «tema social» cuyo desarrollo transcurría en la taberna y en la cárcel; el Madrid que aplaudía en el teatro «Apolo» y desde su estreno en febrero de 1894 «La verbena de la Paloma» o «la Revoltosa», estrenada en el mismo teatro en noviembre de 1897, cuando ya la última de las guerras cubanas entristecía la vida española desde hacía casi dos años. El Madrid en que, junto a informes como los emitidos por la Comisión de Reformas Sociales, pocos han descrito sus contrastes y sus miserias como lo hiciera Pio Baroja en su trilogía "La busca", "Mala hierba» y "Aurora roja», sobre todo al describir sus barrios marginales: «El madrileño que alguna vez, por casualidad, se encuentra en los barrios pobres próximos al Manzanares, hállase sorprendido ante el espectáculo de miseria y sordidez, de tristeza y de incultura que ofrecen las afueras de Madrid con sus rondas miserables, llenas de polvo en verano y de lodo en invierno. La Corte es ciudad de contrastes; presenta luz fuerte al lado de sombra oscura; vida refinada, casi europea, en el centro; vida africana, de aduar, en los suburbios» ${ }^{3}$.

2. Desde hacia dos años, la guerra venía pesando sobre la vida madrileña, especialmente en el ámbito de las familias que no disponían de recursos para pagar la redención en metálico de sus hijos llamados a filas. La primavera de 1898 se manifestó también en la vida económica madrileña en los sobresaltos que el alza de los cambios exteriores produjo entre la clientela de los bancos. Tanto en Madrid como en Barcelona fueron frecuentes, desde mediados de abril, las colas para 
cambiar billetes contra metal. Este miedo se vió también reflejado en el rechazo que algunos establecimientos comerciales determinaron contra los billetes de banco. Eran síntomas, como escribe Carlos Serrano, de un mal más profundo, la baja de la moneda, visible desde años atrás, que evidenciaba el hecho de que «antes de que se hundiera la flota en Cavite, se estaba hundiendo la peseta en Madrid».

Paralelo a ello y no siempre como su consecuencia inmediata fue el fenómeno del alza de los precios, especialmente sensible para los artículos de primera necesidad, como el pan, que originó en las ciudades españolas una serie de motines populares, al grito de "pan barato» y, como tantas veces en la memoria histórica de las ciudades, de «abajo los consumos». Bien es verdad que tal descontento social se hizo más patente en las pequeñas capitales de provincia y en las poblaciones muy dependientes del ámbito rural y se mostró con mucha menor intensidad en los grandes núcleos como Madrid o Barcelona. En el citado artículo, Carlos Serrano los atribuye fundamentalmente a factores de tipo coyuntural, en este caso, la guerra, y otros de tipo estructural, esto es el movimiento decenal, ya subrayado por Nicolás Sánchez Albornoz para la economía española del ochocientos.

Iniciado el conflicto con los Estados Unidos, en realidad último capítulo de una guerra impopular que venía pesando sobre la sociedad española, y de forma más contundente sobre sus sectores más deprimidos, la indiferencia fue, en Madrid como en otras ciudades, la nota dominante. Otra cosa era el entusiasmo más o menos sincero y con fundamento, que mostrasen algunos órganos de la prensa. Contrastaba indudablemente con aquellas manifestaciones callejeras de un patriotismo exaltado cuando las campañas de Africa en la década de los sesenta. Gabriel Maura recordaba que solo «algunos centenares de manifestantes recorrieron las calles y teatros de Madrid en son de jarana nocherniega más vociferadora que imponente; y este holgorio que se prolongó durante una semana, y la mayor procacidad periodística, fueron los únicos síntomas del temple del espíritu público» ${ }^{4}$. Para observadores como Ramiro de Maeztu, la opción del pueblo estaba clara: «Si consultamos las redacciones de los periódicos, no encontramos más que partidarios de la guerra; pero, seamos francos, si consultamos a las clases sociales que envían sus hijos a la guerra, las cuatro partes de España optarán por la paz (...) Cansados de dar sus hijos para defender una isla en la que ni tiene ascensos que conquistar ni empleos que conseguir» 5 .

3. Ha indicado un especialista en la historia del teatro como Andrés Amorós que «no se ha estudiado suficientemente - creo- la huella 
de la crisis noventa y ochista en el mundo de la zarzuela y el género chico». Pero quizá porque sus efectos no fueron tan grandes como los que, en principio e interpretados a un siglo de distancia, cabría atribuirle. Sabemos que, en esos años dramáticos de la guerra, continuaba el éxito que, desde su estreno, venían teniendo obras como «La Verbena de la Paloma», estrenada en 1894. Si alguna obra se puede vincular directamente con el clima patriótico característico de esos años fue la zarzuela «Cádiz», subtitulada «Episodio nacional cómico - líricodramático», estrenada en el teatro Apolo el 20 de noviembre de 1886. Y lo era especialmente por la famosa «marcha» que, del teatro, pasaría a los desfiles militares, a la despedida de las tropas que embarcaban o a cualquier acto que, directa o indirectamente, tuviera relación con la guerra. El crítico teatral «Chispero» lo resumía así: «El éxito de Cádiz fue clamoroso. El libro gustó mucho y la música muchísimo más. El desfile final del primer acto con la briosísima «marcha de Cádiz», que luego fue histórica en los anales españoles, puso al público en pie y la ovación con que se premió la salida a escena, montando brioso corcel blanco, del liberador de Cádiz, enardeció a todos los espectadores, muchos de los cuales enronquecían vitoreando a España» ${ }^{6}$.

Pero, sin duda, el espectáculo que diariamente congregaba a los madrileños de un amplio abanico social era el teatro, dado el número de salas, de muy distinto nivel tanto en instalación como en calidad de las obras ofrecidas, que había en Madrid. En el más representativo de las clases superiores de la ciudad, el Teatro Real, el recuerdo de la guerra estuvo presente en las llamadas "funciones patrióticas», cualquiera fuese la obra representada siempre que la recaudación estuviera destinada a los combatientes que en los dos alejados escenarios de la guerra, el Pacífico y el Caribe, luchaban por España. Casi siempre estas funciones contaron con la presencia de la propia Reina Regente o de otros miembros de la Familia Real, que pagaban su palco con crecidas sumas.

En el amplísimo repertorio que tanto la zarzuela como el «género chico» ofrecían diariamente en Madrid fueron muy varios los espectáculos que, en su argumento central o en escenas ocasionales, estaban dedicadas a la lucha en que España tenía combatientes. Aunque, como hemos indicado, continuaba representándose con mucho éxito una obra estrenada en 1894, "La verbena de la Paloma», dos fueron las obras más significativas de aquel año. Pocas escenas habrán sido a lo largo de los años más representativas de la vuelta a la patria de los combatientes que el coro de los repatriados de "Gigantes y Cabezudos", obra de Miguel Echegaray y de Fernández Caballero. «Por fin te miro, 
Ebro famoso» ha despertado, cuantas veces se ha rememorado la guerra de Cuba, la emoción patriótica de los espectadores. Esta obra, junto a «El santo de la Isidra», de Arniches y López Torregrosa, fueron los estrenos más aplaudidos en el panorama teatral madrileño del 98.

Pero si el teatro, desde las óperas o los conciertos del Real a las salas de barrio donde se daban sainetes o piezas del género chico, fue una de las manifestaciones del impacto de la guerra en la sociedad madrileña, el gran espectáculo era la que se tenía como "fiesta nacional», las corridas de toros. En numerosas ocasiones durante el conflicto colonial se había celebrado «corridas patrióticas» cuya recaudación iba dedicada a los combatientes en Ultramar. Estas, naturalmente, acentuaron su ritmo de celebración a partir de 1896, cuando la lucha y sus dramáticas consecuencias incidieron con mayor rigor sobre la sociedad española. La prensa y, en concreto un periódico, «El Liberal», contribuyó de forma muy activa a tal finalidad de búsqueda de recursos benéficos con destino a los soldados. Destaquemos, por ejemplo, la corrida celebrada el 13 de noviembre de 1896, en que de forma totalmente generosa torearon tres figuras del arte taurino del momento, como «Guerrita», «Bombita» $\mathrm{y}$ «Reverte», mientras que otros dos nombres ya muy famosos, Lagartijo y Frascuelo, ocupaban como asesores el palco presidencial. A tal corrida fueron invitados aquellos soldados «que se encontrasen en Madrid recuperándose de sus heridas en el sanatorio de la Cruz Roja», quienes, terminada la corrida «de pie y al descubierto, se despidieron del público que les saludó con fuertes aplausos y vivas al ejército» 7 . Entre los donativos llegados desde diversos niveles de la sociedad y las instituciones españolas, cabe recordar las tres mil pesetas que, desde su exilio parisino, envió la reina Isabel II. En total, se recaudaron con aquella iniciativa 115.804 pesetas.

Ya en plena guerra con los Estados Unidos, el 12 de mayo, se celebró otra gran corrida, que comenzó a las dos de la tarde pues, a los diez toros que se debían lidiar, se sumaban otros dos de rejones con los que comenzó la fiesta. Diez toreros ocuparon aquella tarde el ruedo: Mazzantini, Valentín Marlis, Guerrita, Torerito, Lagartijillo, Minuto, Reverte, Fuentes, Bombita y Villita. En el palco presidía el conde de Romanones, alcalde de Madrid, y el maestro Federico Chueca dirigía las tres bandas militares que, entre otras composiciones, naturalmente interpretaron la "marcha de Cádiz» ${ }^{8}$. Como era de esperar, los brindis de los toreros mantuvieron también el tono patriótico, del que puede ser ejemplo este: «Brindo por Usía, por el público en general, por el Ejército y la Marina, y por que no quede un yanqui en el universo». La venta de las 13.011 localidades de la plaza dió una cantidad de 
127.289 pesetas, a las que, sumados los donativos entre los que se encontraban las 10.000 pesetas de la Reina Regente María Cristina, ascendió a un total de 250.599 pesetas ${ }^{9}$.

4. Terminada la guerra, quedaba un capítulo especialmente dramático como era el de la repatriación de los miles de combatientes que en los dos escenarios del Atlántico y del Pacífico habían sido los más directos protagonistas del conflicto. Fue un problema que se dejó sentir en todas las tierras de España, sobre todo en aquellas que por sus condiciones económicas sufrieron en su carne el peso de la guerra, al no poder redimir más que a un reducidísimo número de los mozos llamados a filas. Ese había sido uno de los mayores y más dramáticos contrastes entre la España rural y la España urbana, entre las regiones deprimidas, la mayoría, y las social y económicamente más poderosas. Naturalmente el problema se dejó sentir en Madrid, paso obligado de muchos de los combatientes que volvían a sus hogares. El debate en torno a problema tan grave como el de la repatriación tuvo, como era de esperar, su presencia en la vida parlamentaria. Numerosas fueron las voces en torno a cuestión tan candente. Famosa fue, entre otras, la intervención de Vicente Blasco Ibáñez el 6 de septiembre de 1898, aquella que contenía estas palabras: «Es realmente bochornoso y contrista el ánimo con impresión dolorosa el espectáculo que estamos ofreciendo a Europa con el regreso de los soldados repatriados (...) Los trasatlánticos que a nuestras playas los han conducido, no han sido para ellos un buque de la Patria, sino la barca de Caronte, que los conducía a un infierno de miserias, de desvíos y de penalidades (...) Nosotros no cometeremos la injusticia de exigir responsabilidades o de acusar al gobierno por la mortalidad de los repatriados en cuanto es consecuencia de los rigores del clima y de las penalidades de la campaña, pero sí podemos acusarle de imprevisión, de descuido, de olvido de los soldados. ¡Ah, señores ministros! Bien se conoce que la carne de pobre va barata y os importa poco que mueran esos soldados».

No fue Blasco Ibáñez el único que pronunciara duras palabras hacia el gobierno por esta grave cuestión. Otros diputados sumarían su voz a esa denuncia con similares palabras, con frecuencia basadas en testimonios muy vivos. En la sesión del 9 de septiembre, José Canalejas recordaría «el espectáculo de aquella triste mañana en la que un oficial fue con unos cuantos jóvenes famélicos paseando por las calles de Madrid como Cristo camino del Calvario (...) Yo he presenciado uno de esos vergonzosos espectáculos; no encuentran auxilio; están el gobernador militar de Madrid y el capitán general ocupados; no hay un arranque, un impulso para venir en socorro de aquellos desgraciados; 
no hay nadie». Un año después, Rafael Gasset podía afirmar en el Congreso de los Diputados: «Hoy ya nadie se ocupa de los repatriados».

Estas denuncias proliferaron, tanto en la escena parlamentaria como en la prensa, donde se podía leer noticias como ésta que publicaba «El Socialista»: «en las primeras horas de la mañana llegó a la estación del Norte un tren especial conduciendo un convoy de repatriados (...) El espectáculo que ofrecía la Cuesta de San Vicente partía el alma. Infelices soldados caían al suelo por no poder continuar la marcha. La gente madrugadora presenciaba estas escenas con verdadera lástima. No viendo a esos infelices esqueletos no es posible formar un concepto de su estado. A estas horas los ha visto ya mucha gente por esas calles y Madrid ha debido sentir ya toda la influencia del suceso» ${ }^{10}$. Ante comentarios como éste, la medida del gobierno fue la de evitar, en lo posible, el paso de las tropas repatriadas por Madrid y establecer los puntos de trasbordo por ferrocarril en otros nudos ferroviarios. Evitando la visión directa de la tragedia, se evitaba la posible reacción social. Así, como escribía «El Imparcial», «lo que se quiere, sin duda, es que la capital de la monarquía no presencie el triste cuadro que ofrecen esas legiones de desgraciados y no advierta la inhabilidad con que los medios oficiales atienden a la comodidad del triste viajero» ${ }^{11}$.

Entre las páginas tristes de la repatriación se intercalaban otras con homenajes al heroismo demostrado en aquella guerra por hijos del pueblo de Madrid. Quizá su mejor ejemplo fuese el de Eloy Gonzálo, el popular "héroe de Cascorro", cuya estatua, en la plaza donde comienza el Rastro, sigue siendo uno de los enclaves más conocidos y castizos del Madrid de nuestros días. Eloy Gonzálo reunía todos los caracteres de un héroe del pueblo. Origen humilde, depositado en la Inclusa de Madrid y adoptado por un guardia civil destinado en el cercano pueblo de Chapinería. Ingresado en el cuerpo de Carabineros, procesado por insubordinación y amenazas a un superior y condenado a prisión en Valladolid, que èl mismo evitaría cumplir, al amparo del decreto de 25 de agosto de 1895, alistándose en el ejército que combatía en la isla de Cuba, adonde llegaría el 9 de diciembre de ese mismo año, formando parte del primer batallón del regimiento de Infantería «María Cristina», destinado en Puerto Príncipe. La hazaña que le elevaría a la condición de héroe es muy conocida. El 18 de abril de 1896, a la primera compañía de aquel batallón se le encomendaba la protección de Cascorro, una pequeña localidad a 63 kilómetros de Puerto Príncipe, defendido por tres fortines.

El 22 de septiembre de 1896, una ofensiva cubana al mando del comandante general en la provincia de Oriente, Calixto García, con 
cinco mil hombres puso en desesperada situación a los 171 de la posición de Cascorro. Tomada una casa a cincuenta metros del fortín donde estaba Eloy Gonzálo, éste se ofreció a aproximarse a la citada casa para incendiarla, cumpliendo su propósito y volviendo a su posición. En el parte de aquella acción se recordaría que, a petición propia, iba atado a una cuerda para que pudieran «tirar de él y no quedar en poder del enemigo en caso de morir». Defendida heroicamente la posición de Cascorro, sería liberada el 6 de octubre por las tropas al mando del general Jiménez Castellanos, comandante general de Camaguey. La acción de Gonzálo sería recompesada con la cruz de plata del Mérito Militar con distintivo rojo, pensionada con 7,50 pesetas mensuales con caracter vitalicio. Como era muy común en aquella lucha, cuyo principal enemigo eran las enfermedades, Eloy Gonzálo, que había salido ileso de su arriesgada acción, moría el siguiente 18 de junio de una enterocolitis ulcerosa. Repatriados sus restos en el vapor «San Ignacio», el 7 de diciembre de 1898 eran multitudinariamente recibidos en Madrid y llevados a la catedral de La Almudena para posteriormente depositarlos en el mausoleo destinado a los soldados caidos en Cuba y en Filipinas.

Pero, en el conjunto de las páginas dolorosas sigue pesando, como reflexión sobre el postnoventa y ocho, la que Carlos Seco calificaba de «atonía estupefacta de los gritadores de la víspera de la derrota», la de aquellos que fundamentalmente desde la prensa habían animado a la solución bélica, a la que no se pudo resistir el gobierno de Sagasta ${ }^{12}$. No pocos extranjeros, observadores de cómo reaccionaba la sociedad española española, se extrañaban de la indiferencia que se había adueñado de un considerable sector de aquella. Se extrañaban del comentario que en muchas calles de Madrid o de otras ciudades escuchaban sobre la suerte de las colonias: «Es preferible haberlas perdido". Parecía como si ni las derrotas de aquel verano ni las onerosas condiciones de los posteriores tratados de paz hubieran llegado a oídos de muchos españoles. Era la forma de entender la guerra de muchos de aquellos que no la habían tenido como «su guerra», de quienes descansar «era su apetencia más fuerte» ${ }^{13}$.

\section{Notas}

1 ANTONIO FERNÁNDEZ GARCIA, "La población madrileña entre 1876 y 1931. El cambio del modelo demográfico", en La sociedad madrileña durante la Restauración, Madrid, Alfoz, 1989, vol. I, pp. 25-77. 


\section{La sombra del 98}

2 En Manuel Espadas Burgos, "El Madrid de la Restauración», Madrid, Real Sociedad Matritense, 1991, p. 81.

3 Pio BAROJA, «La busca», Madrid, Salvat, 1969, p. 55.

4 En Antonio Fernández García, «Historia y Política», Madrid en Galdós, Galdós en Madrid, Comunidad de Madrid, 1988, t. I, p. 59.

5 RAMiro De MAeZTU, «Hacia otra España», Madrid, Biblioteca Nueva, 1997. Cit. en MARÍa CRUZ SEOANE, "la guerra de 1898 en la prensa española», en "Prensa y opinión en 1898", La Habana, Asociación de Periodistas Europeos, 1999, p. 60.

6 MARÍA Teresa Espín TEMPlado, «El teatro por horas en Madrid (1870-1995)», Madrid, Inst. de Estudios Madrileños, 1995, p. 215.

7 MiguelANGel LÓPEZ RINCONADA, «Las corridas benéfico-patrióticas (1895-1898)», Madrid, 1996, p. 28.

8 ANDRÉS AMORÓS, «Los espectáculos en el 98», en OCTAVIO RUIZ MANJÓN y ALICIA LANFGA (eds.), Los significados del 98, Madrid, Biblioteca Nueva, Universidad Complutense, 1999 , p. 544

9 LÓPEZ RinCONADA, op. cit., p. 55.

10 «El Socialista», 9 septiembre 1898. Cit. en MARÍA ANTONIA FERnÁNDEZ JimÉnez, "La repatriación de los soldados españoles en la prensa del momento", en MiRTA NÚÑEZ Y ANTONIO ROJAS (eds.) «El día después. España y sus excolonias tras el desastre del 98", Madrid, Argés, 1998, p. 162.

11 «El Imparcial», 10 Septiembre 1898.

12 Carlos Seco Serrano, ABC, 13 mayo 1998.

13 Miguel de Unamuno, Obras Completas, Madrid, 1968, t. III, p.831. 